\title{
Efficacy of Dendritic Cell-Based Cancer Immunotherapy
}

Shigetaka Shimodaira ${ }^{1 *}$, Yumiko Higuchi', Ryu Yanagisawa', Masato Okamoto ${ }^{2}$ and Shigeo Koido ${ }^{3}$

${ }^{1}$ Center for Advanced Cell Therapy, Shinshu University Hospital, Matsumoto, Japan

${ }^{2}$ Department of Advanced Immunotherapeutics, Kitasato University School of Pharmacy, Japan

${ }^{3}$ Department of Gastroenterology and Hepatology, The Jikei University School of Medicine, Kashiwa, Japan

\begin{abstract}
Dendritic cells (DCs) have antigen-specific bioactivity against tumor-associated antigens. The acquisition of tumor immunity with DC vaccination could be determined using both tetramer analysis and interferon y-producing clones in enzyme-linked immunosorbent spot assays. A combination of DC vaccination with low-dose metronomic therapy and chemoradiotherapy would contribute to an enhanced acquired immunity for cancer therapy. The efficacy of DC vaccination may provide a survival benefit in some patients with cancer by achieving a prolonged quality-adjusted life year.
\end{abstract}

Keywords: Dendritic cells; Cancer vaccination; Wilms' tumor 1; Metronomic therapy; Quality-adjusted life year

Abbreviations: DCs: Dendritic cells; CTLs: Cytotoxic T Cells; WT1: Wilms' Tumor 1; HLA: Human Leukocyte Antigen; WT1-CTLs: WT1 Antigen-Specific Cytotoxic T Cells; ELISPOT: Enzyme-Linked Immunosorbent Spot

\section{Dendritic Cell-based Cancer Immunotherapy}

Dendritic cells (DCs) may have antigen-specific bioactivity against tumor-associated antigens. Factors affecting the induction of tumor antigen-specific cytotoxic $\mathrm{T}$ cells (CTLs) with DC vaccination remain to be completely elucidated. DC vaccination therapies as an adjuvant to chemo- and/or radiotherapy and the duration for adapting these therapies have been investigated.

Wilms' tumor 1 (WT1) antigen has been reported to be strongly immunogenic [1]; moreover, human leukocyte antigen (HLA)$\mathrm{A}^{\star}$ 24:02-restricted modified WT1 peptide may have considerable efficacy in promoting cancer immunity [2]. DC vaccines primed with WT1-class I/II peptides were safe during chemotherapy for pancreatic cancer [3].

Immunological monitoring of DC vaccination is an important validation tool in clinical studies and trials for proving the concept. WT1 antigen-specific cytotoxic T cells (WT1-CTLs) were determined using both WT1-peptide/HLA-A ${ }^{\star} 24: 02$ tetramer analysis and interferon $\gamma$-producing clones in enzyme-linked immunosorbent spot (ELISPOT) assays during the course of DC vaccination; these analyses are required to be reproducible and validated methods with simple, easily reproducible protocols as shown in Figure 1. The presence of WT1-CTLs in ELISPOT assays was defined according to the following criteria: (1) at least $15 \mathrm{WT} 1$-specific spots per $1 \times 10^{6}$ peripheral blood mononuclear cells and (2) at least a 1.5 -fold increase in the presence of WT1-specific spots compared with peptide spots in the negative control. In contrast, WT1 tetramer-positive CTLs were defined according to the following criteria: (1) comprising at least $0.02 \%$ of $\mathrm{CD}^{+}{ }^{+} \mathrm{CD}^{+}$subset of $50,000-100,000$ lymphocytes and (2) forming a clustered and not diffused population [4]. A positive relationship was determined between the ELISPOT assays and tetramer analysis, and WT1-specific immune responses were detected in $89.1 \%$ of patients with cancer during post-vaccination analysis [4].

Following one course of DC vaccination primed with HLA-A ${ }^{\star} 24: 02-$ restricted-modified WT1 peptide, the tetramer analysis and ELISPOT assays revealed that WT1-CTLs persisted over 1-2 years without any additional DC vaccines [5,6]. Furthermore, the amount of WT1-CTLs markedly increased after a second course of DC vaccination primed with WT1-class I/II peptides, suggesting a boosting reaction and an efficacy of additional reaction with the WT1-class II helper peptide.

\section{Perspectives}

Additional DC vaccinations with chemotherapy and chemoradiotherapy were safe and tolerable, suggesting an enhanced acquired immunity [5]. A low-dose metronomic chemotherapy with cyclophosphamide and/or methotrexate would be a selective combination therapy targeting vascular endothelium [7]. Low-dose metronomic therapy generates tumor adjuvanticity by selective control of regulatory $\mathrm{T}$ cells, increase in natural killer cell activity, and activation of DCs [8-11]. A combination of DC-based adjuvant vaccination and metronomic therapy may contribute to an increase in the efficacy of acquired immunity [6]. Combination therapy with immune checkpoint
Tetramer analysis
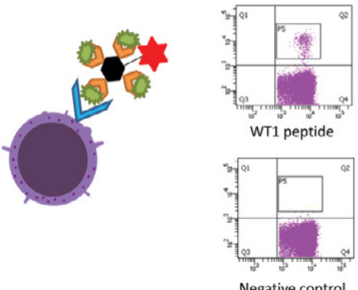

Negative control
ELISPOT assays
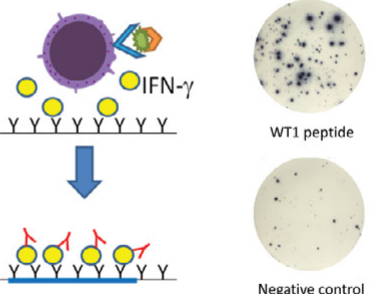

Figure 1: Tetramer analysis and enzyme-linked immunosorbent spot (ELISPOT) assays for detecting Wilms' tumor 1 (WT1)-specific T cells that were induced by dendritic cell vaccination.

*Corresponding author: Shigetaka Shimodaira, Center for Advanced Cell Therapy, Shinshu University Hospital, Matsumoto, Japan, Tel: +81-263-37-3580; Fax: +81-26337-3580; E-mail: shimodai@shinshu-u.ac.jp

Received: January 16, 2016; Accepted: January 25, 2016; Published January 28, 2016

Citation: Shimodaira S, Higuchi Y, Yanagisawa R, Okamoto M, Koido S (2016) Efficacy of Dendritic Cell-Based Cancer Immunotherapy. Biochem Anal Biochem 5: 240. doi:10.4172/2161-1009.1000240

Copyright: (c) 2016 Shimodaira S, et al. This is an open-access article distributed under the terms of the Creative Commons Attribution License, which permits unrestricted use, distribution, and reproduction in any medium, provided the original author and source are credited. 
inhibitors, such as blockade of programmed death 1 and programmed death ligand 1 [12], has the potential to further improve the efficacy of DC vaccination therapies in patients with cancer.

The efficacy of DC vaccination as an adjuvant therapy in some patients with cancer may provide a survival benefit by achieving disease stability and relapse-free survival. In addition to standard cancer therapies, DC vaccination is essentially expected to achieve prolonged quality-adjusted life year, which is a generic measure of both quality and quantity of life under a medical intervention and a measure of the economic value for each individual [13].

\section{Acknowledgment}

This study was supported by the grants-in-aid for scientific research from the Japan Society for the Promotion of Science.

\section{References}

1. Cheever MA, Allison JP, Ferris AS, Finn OJ, Hastings BM, et al. (2009) The prioritization of cancer antigens: a national cancer institute pilot project for the acceleration of translational research. Clin Cancer Res 15: 5323-5337.

2. Sugiyama H (2005) Cancer immunotherapy targeting Wilms' tumor gene WT1 product. Expert Rev Vaccines 4: 503-512.

3. Koido S, Homma S, Okamoto M, Takakura K, Mori M, et al. (2014) Treatment with chemotherapy and dendritic cells pulsed with multiple Wilms' tumor 1 (WT1)-specific MHC class I/II-restricted epitopes for pancreatic cancer. Clin Cancer Res 20: 4228-4239.
4. Higuchi Y, Koya T, Yuzawa M, Yamaoka N, Mizuno Y, et al. (2015) Enzymelinked immunosorbent spot assay for the detection of Wilms' tumor 1-specific T cells induced by dendritic cell vaccination. Biomedicines 3: 304-315.

5. Shimodaira S, Kobayashi T, Hirabayashi K, Horiuchi K, Koya T, et al. (2015) Induction of antigen-specific cytotoxic $\mathrm{T}$ lymphocytes by chemoradiotherapy in patients receiving Wilms' tumor 1-targetted dendritic cell vaccinations for pancreatic cancer. OMICS J Radiol 4: 4

6. Shimodaira S, Sano K, Hirabayashi K, Koya T, Higuchi Y, et al. (2015) Dendritic cell-based adjuvant vaccination targeting Wilms' tumor1 in patients with advanced colorectal cancer. Vaccines 3: 1004-1018

7. Kerbel RS, Kamen BA (2004) The anti-angiogenic basis of metronomic chemotherapy. Nat Rev Cancer 4: 423-436.

8. Lutsiak ME, Semnani RT, De Pascalis R, Kashmiri SV, Schlom J, et al. (2005) Inhibition of CD4(+)25+ T regulatory cell function implicated in enhanced immune response by low-dose cyclophosphamide. Blood 105: 2862-2868

9. André N, Carré M, Pasquier E (2014) Metronomics: towards personalized chemotherapy? Nat Rev Clin Oncol 11: 413-431.

10. Maiti R (2014) Metronomic chemotherapy. J Pharmacol Pharmacother 5: 186-192.

11. Kareva I, Waxman DJ, Lakka Klement G (2015) Metronomic chemotherapy: an attractive alternative to maximum tolerated dose therapy that can activate anti-tumor immunity and minimize therapeutic resistance. Cancer Lett 358: 100-106.

12. Shih K, Arkenau HT, Infante JR (2014) Clinical impact of checkpoint inhibitors as novel cancer therapies. Drugs 74: 1993-2013.

13. QALY. 